\title{
Study of X-cor Sandwich's Compression Modulus Based on Homogenization Theory
}

\author{
Xu-dan Dang ${ }^{\mathrm{a} 1,4,5, *}$, Shao-jie Shi ${ }^{\mathrm{b} 2}$, Jun Xiao ${ }^{\mathrm{c} 3}$, Bing-bing Fan ${ }^{\mathrm{d} 4, *}$, Wei-min \\ Long $^{\mathrm{e}}$, Rui Zhang ${ }^{\mathrm{f4}, 6, *}$ and Lin-jun $\mathrm{Li}^{\mathrm{d} 1}$
}

\author{
${ }^{1}$ School of Mechanical Engineering, Henan University of Engineering, Zhengzhou 450007, China \\ ${ }^{2}$ Personnel department, Henan University of Engineering, Zhengzhou 450007, China \\ ${ }^{3}$ College of Materials Science and Technology, Nanjing University of Aeronautics and Astronautics, \\ Nanjing 210016, China \\ ${ }^{4}$ School of Materials Science and Engineering, Zhengzhou University, Zhengzhou 450001, China \\ ${ }^{5}$ Zhengzhou Research Institute of Mechanical Engineering, Zhengzhou 450001, China \\ ${ }^{6}$ Provincial Key Laboratory of Aviation Materials and Application Technology, Zhengzhou University \\ of Aeronautics, Zhengzhou 450015, China

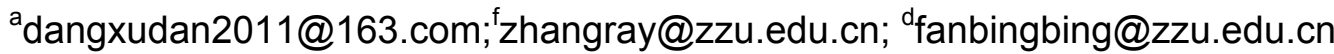 \\ ${ }^{*}$ Corresponding author
}

Keywords: X-cor Sandwich, Z-pin, Homogenization Theory, Compression Modulus.

\begin{abstract}
As new structural composites, X-cor sandwich effectively improves the mechanical properties of the foam sandwich in thickness direction due to its high specific strength and specific stiffness. In this article, by introducing the correction coefficient, the modified calculation model of X-cor sandwich's compression modulus was established based on homogenization theory. The correction coefficient of compression modulus is 0.3 . The effects of X-cor sandwich's structural parameters on the compression modulus were investigated. In Z-pin's inserting angle range of $0^{\circ}-30^{\circ}$, the compression modulus decreases with the increase of inserting angle and increases with the increase of Z-pin's density and diameter. The error range between the analysis of modified model and the test is $-13.9 \%-10.6 \%$. The analysis is in good agreement with the test, which indicates that the modified calculation model can be used to calculate the X-cor sandwich's compression modulus.
\end{abstract}

\section{Introduction}

As a kind of lightweight structure, the sandwich is composed of facesheets, cores and glued layers [1]. The facesheet is made of high strength and high modulus materials. The core can be made using honeycomb, foam and other lightweight materials. One of the problems of honeycomb sandwich is its complex preparation process. Furthermore, there easily appear pits at the location of honeycomb core on the facesheet, which will severely affect the smooth flatness of facesheet [2]. This problem can be effectively solved by using the closed cell rigid foam as cores. In the X-cor sandwich, numerous unidirectional composite needles (Z-pin) with high strength and high modulus were inserted into the foam in a certain degree to form $X$ shape spatial truss structure. The prepreg were laid on the foam core containing the truss structure. After the compound curing, the tips of Z-pin inserted into the facesheets to form a kind of integral structure, resulting in the improvements of modulus and strength in thickness direction [3-5]. The novel structural composites well satisfy the requirements for light weight and high strength of the materials for high technology, such as aeronautics and astronautics, etc.

At present, the X-cor sandwich's preparation, microstructure, calculations of the lastic and strength properties and failure mechanism are deeply studied abroad [6-9]. While the related researches about $\mathrm{X}$-cor sandwich start later in China. It is at the stage of exploration and the X-cor sandwich's 
mechanical model is especially incomplete [10-13]. In this study the calculation model of X-cor sandwich's compression modulus is modified based on homogenization theory. The rationality of the modified model is verified through the comparison of calculated values and test values.

\section{Preparation and Tests of X-cor Sandwich}

The preparation of X-cor sandwich is composed of three steps. The first step is Z-pin's pultrusion. The fibers used in Z-pin's pultrusion were carbon fibers (Toray, Japan, T300-3K and T300-6K) and glass fibers (Nanjing Glass Fiber Research Institute, HS4). The resin system was FW-63 epoxy resin (Yubo Company, Kunshan). Figure 1 exhibits the pultrusion Z-pins of carbon fiber/FW-63 epoxy resin. The second step is Z-pin's insertion. The pultrusion Z-pins were inserted into the foam cores by the implanting machine to form the reinforced foam. The elastic modulus of PMI foam (Degussa, Germany, Rohacell 31IG) was $36 \mathrm{MPa}$. Figure 2 shows the X-cor after Z-pin's insertion.

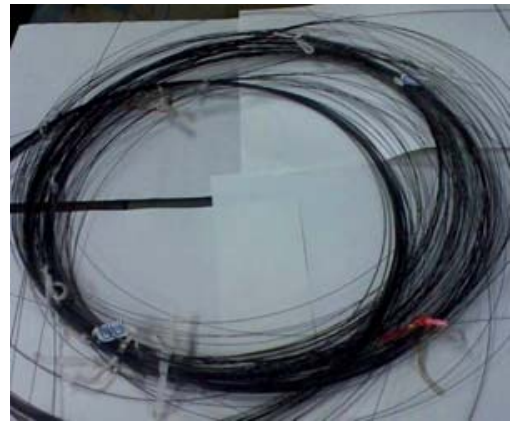

Figure 1. Pultrusion Z-pins

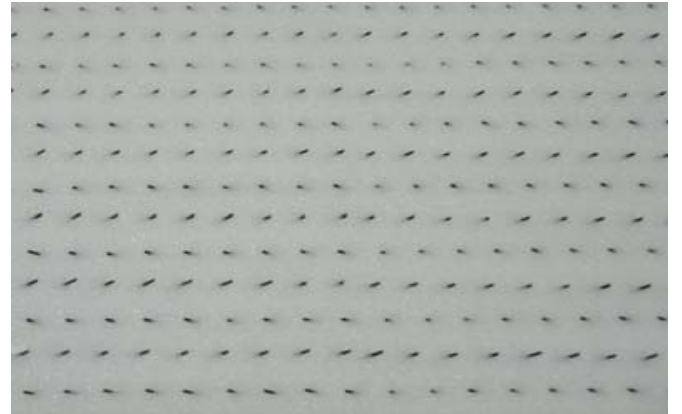

Figure 2. X-cor (reinforced foam)

The third step is the curing of X-cor sandwich using vacuum solidification forming process. Figure 3 and 4 are the vacuum bag curing device and the X-cor sandwich, respectively.

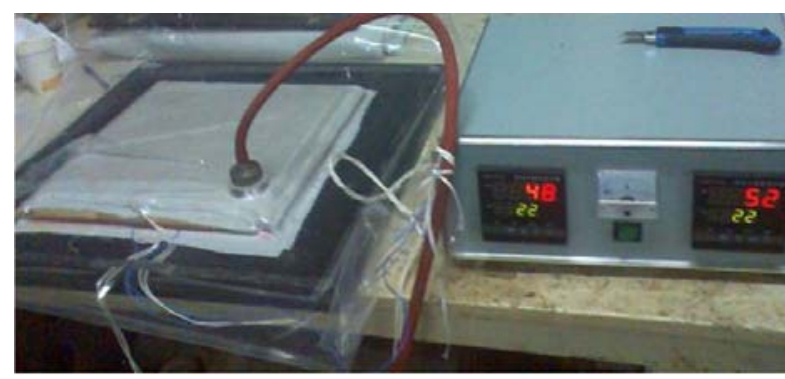

Figure 3. Vacuum bag curing device

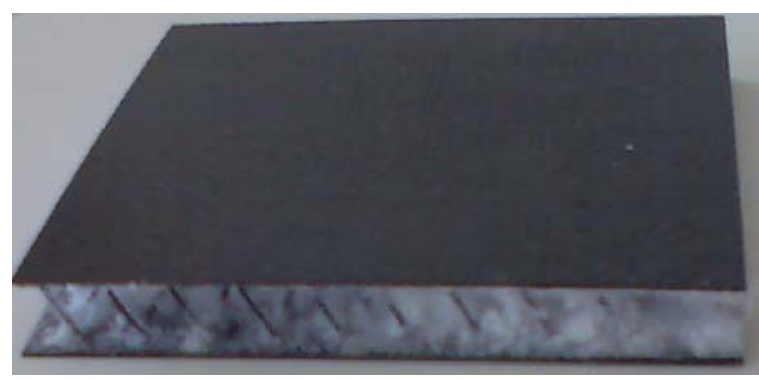

Figure 4. X-cor sandwich

\section{Establishment of Modified Model}

Z-pins in the X-cor sandwich preserve the feature of periodic distribution and the mechanical properties of the sandwich can be adjusted through changing Z-pins' geometric parameters. The homogenization theory offers an effective way to combine the macro and micro level of materials [15]. The calculation model of X-cor sandwich's compression modulus was established based on homogenization theory. The equivalent stiffness tensor $C^{H}$ of the X-cor sandwich is Equation 1 [8]. 


$$
\begin{aligned}
C^{H}=\frac{2 \cdot \pi \cdot r^{2} \cdot E_{\mathrm{p}}}{h \cdot D_{2} \cdot D_{3} \cdot\left(D_{1}^{2}+h^{2}\right)^{3 / 2}}\left[\begin{array}{lllllll}
D_{1}^{4} & 0 & h^{2} \cdot D_{1}^{2} & 0 & 0 & 0 \\
0 & D_{1}^{4} & h^{2} \cdot D_{1}^{2} & 0 & 0 & 0 \\
h^{2} \cdot D_{1}^{2} & h^{2} \cdot D_{1}^{2} & 2 \cdot h^{4} & 0 & 0 & 0 \\
0 & 0 & 0 & h^{2} \cdot D_{1}^{2} & 0 & 0 \\
0 & 0 & 0 & 0 & h^{2} \cdot D_{1}^{2} & 0 \\
0 & 0 & 0 & 0 & 0 & 0
\end{array}\right] \\
+\left(1-V_{\mathrm{p}}\right) \cdot \frac{E_{\mathrm{f}} \cdot\left(1-v_{\mathrm{f}}\right)}{\left(1+v_{\mathrm{f}}\right) \cdot\left(1-2 \cdot v_{\mathrm{f}}\right)}\left[\begin{array}{llllllll}
\frac{v_{\mathrm{f}}}{1} & \frac{v_{\mathrm{f}}}{\left(1-v_{\mathrm{f}}\right)} & \frac{v_{\mathrm{f}}}{\left(1-v_{\mathrm{f}}\right)} & 0 & 0 & 0 \\
\frac{v_{\mathrm{f}}}{\left(1-v_{\mathrm{f}}\right)} & 1 & \frac{v_{\mathrm{f}}}{\left(1-v_{\mathrm{f}}\right)} & \frac{1}{\left(1-v_{\mathrm{f}}\right)} & 0 & 0 & 0 & 0 \\
0 & 0 & 0 & \frac{\left(1-2 \cdot v_{\mathrm{f}}\right)}{2 \cdot\left(1-v_{\mathrm{f}}\right)} & 0 & 0 \\
0 & 0 & 0 & 0 & \frac{\left(1-2 \cdot v_{\mathrm{f}}\right)}{2 \cdot\left(1-v_{\mathrm{f}}\right)} & 0 \\
0 & 0 & 0 & 0 & 0 & \frac{\left(1-2 \cdot v_{\mathrm{f}}\right)}{2 \cdot\left(1-v_{\mathrm{f}}\right)}
\end{array}\right]
\end{aligned}
$$

In Eq.1, $D_{1}, D_{2}$ and $D_{3}$ are the Z-pin's projection length at the bottom surface of cell, the cell length and width, respectively; $E_{\mathrm{p}}$ and $E_{\mathrm{f}}$ are the elastic modulus of Z-pin and foam, respectively; $v_{\mathrm{f}}$ is the Poisson ration of foam. The first item on the right in Eq.1 is the Z-pin's contribution to stiffness and the second item is the contribution of foam. The followed relationship can also be achieved through the X-cor sandwich's cell (Eq. 2).

$$
\cos \omega=\frac{h}{\left(D_{1}^{2}+h^{2}\right)^{1 / 2}}, \sin \omega=\frac{D_{3}}{\left(D_{1}^{2}+h^{2}\right)^{1 / 2}}
$$

The X-cor sandwich's stiffness in thickness direction $C_{33}^{H}$ is Equation 3.

$$
C_{33}^{H}=\frac{2 \cdot \pi \cdot r^{2} \cdot E_{\mathrm{p}}}{h \cdot D_{2} \cdot D_{3} \cdot\left(D_{1}^{2}+h^{2}\right)^{3 / 2}} \cdot 2 \cdot h^{4}+\left(1-V_{\mathrm{p}}\right) \cdot \frac{E_{\mathrm{f}} \cdot\left(1-v_{\mathrm{f}}\right)}{\left(1+v_{\mathrm{f}}\right) \cdot\left(1-2 \cdot v_{\mathrm{f}}\right)}
$$

Equation 4 can be achieved through the combination of Eq. 2- 3.

$$
C_{33}^{H}=\frac{4 \cdot \pi \cdot r^{2} \cdot E_{\mathrm{p}}}{D_{2} \cdot D_{3}} \cdot \cos ^{3} \omega+\left(1-V_{\mathrm{p}}\right) \cdot \frac{E_{\mathrm{f}} \cdot\left(1-v_{\mathrm{f}}\right)}{\left(1+v_{\mathrm{f}}\right) \cdot\left(1-2 \cdot v_{\mathrm{f}}\right)}
$$

According to Eq. 1-4, in this study there are two Z-pins in cell, so $C_{33}^{H}$ is expressed as Equation 5.

$$
C_{33}^{H}=\frac{2 \cdot \pi \cdot r^{2} \cdot E_{\mathrm{p}}}{D_{2} \cdot D_{3}} \cdot \cos ^{3} \omega+\left(1-V_{\mathrm{p}}\right) \cdot \frac{E_{\mathrm{f}} \cdot\left(1-v_{\mathrm{f}}\right)}{\left(1+v_{\mathrm{f}}\right) \cdot\left(1-2 \cdot v_{\mathrm{f}}\right)}
$$


Eq. 1 shows the stiffness matrix of the X-cor sandwich. The stiffness matrix inversion (flexibility matrix) can be achieved by the calculation $\left([S]=[C]^{-1}\right)$. Therefore, the X-cor sandwich's compression modulus in thickness direction can be calculated $\left(E_{\mathrm{c}}=1 / S_{z z}\right)$. The expression of $E_{\mathrm{c}}$ is Equation 6 .

$$
E_{\mathrm{c}}=\frac{\left(1-v_{\mathrm{yx}} \cdot v_{\mathrm{xy}}-v_{\mathrm{zy}} \cdot v_{\mathrm{yz}}-v_{\mathrm{xz}} \cdot v_{\mathrm{zx}}-2 \cdot v_{\mathrm{xy}} \cdot v_{\mathrm{yz}} \cdot v_{\mathrm{zx}}\right)}{\left(1-v_{\mathrm{yx}} \cdot v_{\mathrm{xy}}\right)} \cdot C_{33}^{H}
$$

In Eq. 6, items on the left of $C_{33}^{H}$ are composed of Poisson ratios of the X-cor sandwich and the coefficient is approximately 0.74 . However, Eq. 6 indicates a kind of ideal case. In practical use, the predicted valus are larger than the test valus. In the X-cor sandwich, there sometims appear defects due to the process or other reasons. Thus the compression modulus does not reach the theoretical values. Therefore, the model of X-cor sandwich's compression modulus is modified by introducing the correction coefficient in order to solve this problem. The correction coefficient of compression modulus is named Mofidication of the Compression Modulus (Mcm) and its value here is 0.3. The introduction of correction coefficient reflects the effects of process on the X-cor sandwich's properties. Equation 7 expresses the modified calculation model.

$$
E_{\mathrm{c}}=M_{\mathrm{cm}} \cdot 0.74 \cdot\left(\frac{2 \cdot \pi \cdot r^{2} \cdot E_{\mathrm{p}}}{D_{2} \cdot D_{3}} \cdot \cos ^{3} \omega+\left(1-V_{\mathrm{p}}\right) \cdot \frac{E_{\mathrm{f}} \cdot\left(1-v_{\mathrm{f}}\right)}{\left(1+v_{\mathrm{f}}\right) \cdot\left(1-2 \cdot v_{\mathrm{f}}\right.}\right)
$$

The comparison of calculated values and test values of X-cor sandwich's compression modulus is listed in Table 1. In table 1, there were three types of X-cor sandwich, which were carbon fiber Z-pins-carbon fiber facesheets (CF-CF), glass fiber Z-pins-glass fiber facesheets (GF-GF) and carbon fiber Z-pins-glass fiber facesheets (CF-GF).

Table 1. Comparison of calculated values and test values of compression modulus

\begin{tabular}{|c|c|c|c|c|c|c|}
\hline X-cor type & Distance/[mm] & Diameter/[mm] & angle/ $\left[{ }^{\circ}\right]$ & Test/[MPa] & Calculation/[MPa] & Error/[\%] \\
\hline CF-CF & $5 \times 10$ & 0.5 & 30 & 77.6 & 85.8 & 10.6 \\
\hline CF-CF & $5 \times 10$ & 0.5 & 22 & 93.1 & 102.8 & 10.4 \\
\hline CF-CF & $5 \times 10$ & 0.5 & 15 & 106.4 & 114.7 & 7.8 \\
\hline CF-CF & $5 \times 10$ & 0.5 & 0 & 118.9 & 126.3 & 6.2 \\
\hline GF-GF & $5 \times 10$ & 0.7 & 22 & 108.9 & 93.8 & -13.9 \\
\hline GF-GF & $5 \times 10$ & 0.7 & 15 & 113.8 & 104.6 & -8.1 \\
\hline GF-GF & $5 \times 10$ & 0.7 & 0 & 116.8 & 114.9 & -1.6 \\
\hline GF-GF & $5 \times 5$ & 0.7 & 22 & 178.7 & 176.8 & -1.1 \\
\hline GF-GF & $5 \times 5$ & 0.5 & 15 & 106.2 & 106.5 & 0.3 \\
\hline CF-GF & $10 \times 10$ & 0.5 & 30 & 45.5 & 48.3 & 6.2 \\
\hline CF-GF & $5 \times 5$ & 0.5 & 22 & 185.5 & 194.9 & 5.1 \\
\hline
\end{tabular}

The calculated values of homogenization theory agree well with the tested values (Table 1), which indicates that the introduction of correction coefficient is reasonable. Therefore, the modified model based on homogenization theory can predict the X-cor sandwich's compression modulus.

\section{Conclusions}

The following conclusions can be drawn through investigations of the X-cor sandwich's compression modulus. The modified calculation model of X-cor sandwich's compression modulus was established based on homogenization theory and the correction coefficient is 0.3 . Through the calculation of modified model, the effects of structural parameters on X-cor sandwich's compression modulus were achieved. In the Z-pin's insertion angle range of $0^{\circ}-30^{\circ}$, the compression modulus 
decreases with the increase of insertion angle and increases with the increase of Z-pin's density and diameter. The error range between calculated values and test values is $-13.9 \%-10.6 \%$. The agreement indicates the rationality and correctness of the modified calculation model.

\section{Acknowledgement}

This research was financially supported by the National Natural Science Foundation of China (Grant No. 51172213, 50972132 and U1304512), Natural Science Research Project of Henan Educational Committee (Grant No. 15A430018). The authors would like to thank for the support.

\section{References}

[1] Y. Shen, Y. Li, X. Wang, D. Huan, Low-velocity impact effect on the mechanical properties of K-cor sandwich structure, Acta Aeronautica et Astronautica Sinica. 35 (2014) 3853-3863.

[2] T. Huang, A study on mechanical properties of stitching foam-core sandwich, First ed., Xi'an, Shanxi, 2004.

[3] T.C. Carstensen, E. Kunkel, C. Magee, X-cor ${ }^{\mathrm{TM}}$ advanced sandwich core material, International SAMPE Technical Conference Series, CA: Society of Advancement Material and Process Engineering, (2001) 452-466.

[4] X. Dang, J. Xiao, Y. Li, Progress of experimental study on mechanical properties of X-cor sandwich structure, J. Mater. Engineering. (6) (2008) 76-80.

[5] D.D.R. Cartié, M. Troulis, I.K. Partridge, Delamination of Z-pinned carbon fibre reinforced laminates, Compos. Sci. Technol. 66 (2006) 855-861.

[6] A.P. Mouritz, Compression properties of z-pinned sandwich composites, J. Mater. Sci. 41 (2006) 5771-5774.

[7] A.P. Mouritz, B.N. Cox, A mechanistic approach to the properties of stitched laminates, Composites Part A Appl. Sci. Manuf. (2000) 1-27.

[8] T. Liu, Z. Deng, T. Lu, Analytical modeling and finite element simulation of the plastic collapse of sandwich beams with pin-reinforced foam cores, Int. J. Solids. Struct. 45 (2008) 5127-5151.

[9] T. Liu, Z. Deng, T. Lu, Design optimization of truss-cored sandwiches with homogenization, Int. J. Solids. Struct. 43 (2006) 7891-7918.

[10]H. Chen, Research on mechanical behavior of X-cor reinforced foam sandwich composited structure, First ed., Shanghai, 2010.

[11] X. Zhang, Y. Li, J. Li, L. Fan, Y. Tan, J. Xiao, Prediction of compressive and shear moduli of $\mathrm{X}$-cor sandwich structures for aeronautic engineering, Transactions of Nanjing University of Aeronautics and Astronautics. 32 (2015) 646-653.

[12]H. Shan, J. Xiao, N. Li, W. Shang, X. Zhang, Experiment and analysis on shear modulus of X-cor sandwich, J. Mater. Engineering. (5) (2014) 59-65.

[13]J. Hao, Z. Zhang, L. Zhang, M. Li, Zh. Sun, Effects of Z-pin inserting parameters on X-cor sandwich mechanical property, Acta Aeronautica et Astronautica Sinica. 29 (2008) 763-768.

[14] Secretariat of national standardization technical committee for fiber reinforced plastics, Standard specification for fiber reinforced plastics (FRP), Second ed., Beijing, 2009.

[15] Y. Xia, Q. Wang, H. Liao, Equivalent models for corrugated panels based on periodic homogenization theory, Sichuan Building Science. 41 (2015) 6-11. 